\title{
WEAKLY CONVEX AND CONVEX DOMINATION NUMBERS FOR GENERALIZED PETERSEN AND FLOWER SNARK GRAPHS
}

\author{
JOZEF KRATICA, DRAGAN MATIĆ, AND VLADIMIR FILIPOVIĆ
}

\begin{abstract}
We consider the weakly convex and convex domination numbers for two classes of graphs: generalized Petersen graphs and flower snark graphs. For a given generalized Petersen graph $G P(n, k)$, we prove that if $k=1$ and $n \geq 4$ then both the weakly convex domination number $\gamma_{w c o n}(G P(n, k))$ and the convex domination number $\gamma_{c o n}(G P(n, k))$ are equal to $n$. For $k \geq 2$ and $n \geq 13, \gamma_{w c o n}(G P(n, k))=\gamma_{c o n}(G P(n, k))=2 n$, which is the order of $G P(n, k)$. Special cases for smaller graphs are solved by the exact method. For a flower snark graph $J_{n}$, where $n$ is odd and $n \geq 5$, we prove that $\gamma_{w c o n}\left(J_{n}\right)=$ $2 n$ and $\gamma_{c o n}\left(J_{n}\right)=4 n$.
\end{abstract}

\section{INTRODUCTION}

Let $G=(V, E)$ be a connected undirected graph without loops and parallel edges. Let $d_{G}(u, v)$ denote the distance between the vertices $u$ and $v$, i.e. the length of a shortest $u-v$ path in $G$. By $\mathcal{N}(v)$ we denote the set of all vertices adjacent to $v$. Let $\mathcal{N}[v]=\mathcal{N}(v) \cup\{v\}$. A set $V^{\prime} \subseteq V$ is a dominating set in $G$ if every vertex $u \in V \backslash V^{\prime}$ is adjacent to a vertex $v \in V^{\prime}$. In other words, a set $V^{\prime} \subseteq V$ is a dominating set in $G$ if for each vertex $v \in V$, it holds that $v \in V^{\prime}$ or $v \in \mathcal{N}(u)$ for some vertex $u \in V^{\prime}$. The domination number (usually denoted by $\gamma(G)$ ) is defined as the minimum cardinality among all dominating sets of $G$. The terms dominating set and domination number of a graph $G$ were first defined by O. Ore in 1962 [28]. A vertex $v \in V$ is dominated by a set $S \subseteq V$ if either $v$ itself or one of its neighbors is in $S$. Many variants of graph domination numbers have been investigated for numerous classes of graphs. In this paper we study the weakly convex domination number and convex domination number of generalized Petersen graphs and flower snark graphs.

2010 Mathematics Subject Classification. 05C59, 68R10.

Key words and phrases. graph domination; convex domination number; weakly convex domination number; generalized Petersen graphs; flower snark graphs.

For the first and third authors, this research was partially supported by the Serbian Ministry of Education and Science under projects 174010 and 174033, while for the second author it was partially supported by the Ministry of Science and Technology of Republika Srpska. 
A vertex set $X \subseteq V$ is a weakly convex set if for every two vertices $u$ and $v$ from $X$, it holds that $d(u, v)$ in $\langle X\rangle$ is equal to $d(u, v)$ in $G$, where $\langle X\rangle$ is a subgraph of $G$ induced by $X$. Equivalently, a vertex set $X \subseteq V$ is a weakly convex set in $G$ if for every two vertices $u, v \in X$, there exists at least one shortest $u-v$ path (in $G$ ), whose vertices belong to $X$. A set $X$ is a weakly convex dominating set if it is weakly convex and dominating. The weakly convex domination number of a graph $G$, denoted by $\gamma_{w c o n}(G)$, is the smallest cardinality of a weakly convex dominating set in $G$. The weakly convex dominating set problem (WCDSP) is therefore defined as the problem of determining a weakly convex dominating set of the smallest cardinality. Here we notice a useful property of weakly convex sets.

Property 1.1. Let $X$ be a weakly convex set and let $u \in X$. If for an arbitrary vertex $v$, the unique shortest path in $G$ between vertices $u$ and $v$ contains vertex $w$, such that $w \notin X$, then $v \notin X$.

A vertex set $X \subseteq V$ is a convex set if for every two vertices $u$ and $v$ from $X$, every shortest $u-v$ path (in $G$ ) also belongs to $\langle X\rangle$. A set $X$ is a convex dominating set if it is convex and dominating. The convex domination number of a graph $G$, denoted by $\gamma_{c o n}(G)$, is the smallest cardinality of a convex dominating set in $G$. The convex dominating set problem (CDSP) is therefore defined as the problem of determining a convex dominating set of the smallest cardinality.

Example 1.2. For the Petersen graph (or generalized Petersen graph $G P(5,2)$ ) given in Figure 11 the solution of both the WCDSP and the CDSP is the set $S=\left\{u_{1}, v_{1}, v_{3}, v_{4}\right\}$, so $\gamma_{w c o n}(G P(5,2))=\gamma_{\text {con }}(G P(5,2))=4$.

For $G P(10,2)$ (given in Figure 2), one can conclude that the set $\left\{u_{i} \mid 0 \leq i \leq 9\right\}$ is a weakly convex dominating set of the smallest cardinality, so $\gamma_{w c o n}(G P(10,2))=$ 10 . By the exact method it is calculated that $\gamma_{\text {con }}(G P(10,2))=20$, which means that all vertices must be included in the minimal convex dominating set.

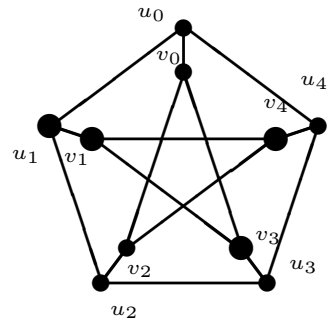

Figure 1. Petersen graph

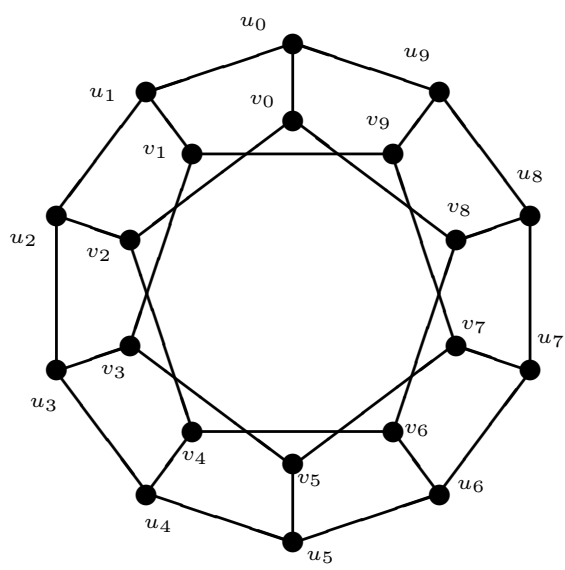

Figure 2. Generalized Petersen graph $G P(10,2)$ 
1.1. Previous work. The convex domination number was firstly introduced in 2002 by Jerzy Topp (Gdansk University of Technology) in personal communication with some other researchers. The decision problems of WCDSP and CDSP are proved to be NP-complete even for bipartite and split graphs [29], so the problems of finding the weakly convex dominating and convex dominating sets of minimal cardinality are NP-hard in a general case.

Relations between $\gamma_{w c o n}$ and $\gamma_{c o n}$ have been studied in [22], where some classes of cubic graphs for which the convex domination number is equal to the domination number have been analysed. Since every convex dominating set is a weakly convex dominating set and every weakly convex dominating set is a dominating set, the following lemma from 22 is obvious:

Lemma $1.3([22])$. For any connected graph $G$,

$$
\gamma(G) \leq \gamma_{w c o n}(G) \leq \gamma_{\text {con }}(G)
$$

In a comprehensive study regarding graphs with convex domination number close to their order [8], a dozen of very useful results have been presented. Among all of them, we emphasize a remark and two theorems, which are of interest for our paper.

Remark 1.4 ([8]). For a cycle $C_{n}$ with $n \geq 6$ vertices, $\gamma_{c o n}\left(C_{n}\right)=n$.

Theorem $1.5([])$. If $G=(V, E)$ is a connected graph with $\delta(G) \geq 2$ and $g(G) \geq$ 6 , then $\gamma_{\text {con }}(G)=|V|$, where $\delta(G)$ is the minimum vertex degree and $g(G)$ is the length of a shortest cycle in $G$.

Theorem $1.6([8])$. For any connected graphs $G$ and $H$, the inequality

$$
\gamma_{\text {con }}(G) \gamma_{\text {con }}(H) \leq \gamma_{\text {con }}(G \square H)
$$

holds, where $G \square H$ denotes the Cartesian product of the graphs $G$ and $H$.

Closed formulas for weakly convex and convex domination numbers of a torus are presented in [30].

The influence of the edge subdivision on the convex domination number is discussed in [9]. In that paper, it is shown that in the general case the convex domination number can be arbitrarily increased and arbitrarily decreased by an edge subdivision.

Convex domination in the Cartesian product and other graph compositions are also studied in [21] and [20]. In [20] closed formulas for weakly convex and convex domination numbers in a grid graph are given. The following result from 21] will be used in Section 2 ,

Theorem 1.7 ([21]). Let $G$ and $H$ be connected graphs of orders $m$ and $n$, respectively. Then $\gamma_{c o n}(G \square H)=\min \left\{m \gamma_{c o n}(H), n \gamma_{c o n}(G)\right\}$.

Nordhaus-Gaddum type results for the weakly convex domination number have been studied in $[23$, and for the convex domination number in 24].

In the following two subsections we shortly explain generalized Petersen graphs and flower snark graphs. 
1.2. Generalized Petersen graphs. The generalization of the Petersen graph was introduced by Coxeter in 1950 [7]. For given numbers $n \geq 3$ and $1 \leq k<$ $n / 2$, the generalized Petersen graph $G P(n, k)$ contains $2 n$ vertices and $3 n$ edges, with the set of vertices $V=\left\{u_{i}, v_{i} \mid 0 \leq i \leq n-1\right\}$ and the set of edges $E=$ $\left\{\left\{u_{i}, u_{i+1}\right\},\left\{u_{i}, v_{i}\right\},\left\{v_{i}, v_{i+k}\right\} \mid 0 \leq i \leq n-1\right\}$, where the subscripts are taken modulo $n$, as we will be doing hereafter. The Petersen graph from Example 1.2 is $G P(5,2)$.

In order to shorten the writing, the set $\left\{u_{0}, \ldots, u_{n-1}\right\}$ will henceforth be called $u$ vertices, and analogously the set $\left\{v_{0}, \ldots, v_{n-1}\right\}$ will be called $v$ vertices. If $k=1$, it is obvious that the sets of $u$ and $v$ vertices have the same meaning, since there is an isomorphism which maps $u$ vertices to $v$ vertices.

In the recently published literature, various properties of $G P(n, k)$ have been investigated: minimum span of $L(2,1)$-labeling [1, minimum vertex cover [4, metric dimension [2, 27], strong metric dimension [18], decycling number [13], component connectivity [10], acyclic 3-coloring [34], crossing numbers [25], independence number [11], and others. Some recent works dealing with variants of the domination numbers in the generalized Petersen graphs are: domination number [3, 12, 26], domatic number, total domatic number, and $k$-ply domatic number [33], efficient domination number [17, power domination number [32, 2-rainbow domination [5, 31, and others.

The following remark will be used in the rest of the paper.

Remark 1.8. $G P(n, 1) \cong C_{n} \square P_{2}$, where $P_{2}$ is the path with two vertices.

1.3. Flower snark graphs. Flower snark graphs appeared in an early work [16] as an example of a cubic bridgeless graph family that is not Tait colorable, i.e. it is not 3-edge colorable. The flower snark $J_{n}$, where $n$ is odd, can be constructed with the following process:

Construct $n$ copies of the star graph of 4 vertices. Let the central vertex of each star be denoted by $a_{i}$ and the outer vertices by $b_{i}, c_{i}$, and $d_{i}$. The union of all these stars is a disconnected graph containing $4 n$ vertices and $3 n$ edges: $a_{i} b_{i}, a_{i} c_{i}$, and $a_{i} d_{i}$ for each $i, 0 \leq i \leq n-1$. We further add $n$ more edges by constructing the cycle $b_{0}, b_{1}, \ldots, b_{n-1}, b_{0}$ of length $n$. Finally, we construct the cycle $c_{0}, c_{1}, \ldots, c_{n-1}, d_{0}, d_{1}, \ldots, d_{n-1}, c_{0}$ of length $2 n$. The graph obtained, denoted by $J_{n}$, is the flower snark graph. This graph is a cubic graph with $4 n$ vertices and $6 n$ edges.

The sets $\left\{a_{0}, \ldots, a_{n-1}\right\},\left\{b_{0}, \ldots, b_{n-1}\right\},\left\{c_{0}, \ldots, c_{n-1}\right\}$, and $\left\{d_{0}, \ldots, d_{n-1}\right\}$ will be respectively called $a, b, c$, and $d$ vertices.

Unlike generalized Petersen graphs, domination numbers, as well as other graph invariants, have not been so intensively studied for flower snarks. We mention some recent works on this class: the circular chromatic index has been studied in [14, the total-chromatic number in [6], and prime cordial labeling in [15].

In the following property, we ensure that for each $j, 0 \leq j \leq n-1$, there exists an isomorphism of $J_{n}$ which maps vertices $b_{j}$ and $a_{j}$ to $b_{0}$ and $a_{0}$, respectively. 
Property 1.9. Let $J_{n}$ be a flower snark and let $j \in\{0,1, \ldots, n-1\}$ be an arbitrary number. Then the function $g: V\left(J_{n}\right) \rightarrow V\left(J_{n}\right)$ defined as

$$
\begin{aligned}
& g\left(a_{i}\right)= \begin{cases}a_{j-i}, & i \leq j \leq n-1, \\
a_{n+j-i}, & 0 \leq j<i,\end{cases} \\
& g\left(b_{i}\right)= \begin{cases}b_{j-i}, & i \leq j \leq n-1, \\
b_{n+j-i}, & 0 \leq j<i,\end{cases} \\
& g\left(c_{i}\right)= \begin{cases}c_{j-i}, & i \leq j \leq n-1, \\
d_{n+j-i}, & 0 \leq j<i,\end{cases} \\
& g\left(d_{i}\right)= \begin{cases}d_{j-i}, & i \leq j \leq n-1, \\
c_{n+j-i}, & 0 \leq j<i,\end{cases}
\end{aligned}
$$

is an isomorphism.

From this, we can obtain another two useful properties which will be referred to later.

Property 1.10. For each $j, 0 \leq j \leq n-1$, without loss of generality the vertices $b_{j}$ and $a_{j}$ can be declared as $b_{0}$ and $a_{0}$.

Property 1.11. For $j=0$, by the function $g$, the 4 -tuple $\left(a_{0}, b_{0}, c_{0}, d_{0}\right)$ maps to itself, while for $0<i \leq n-1$, the 4 -tuple $\left(a_{i}, b_{i}, c_{i}, d_{i}\right)$ maps to $\left(a_{n-i}, b_{n-i}, d_{n-i}, c_{n-i}\right)$.

We also notice a useful property regarding the shortest paths in $J_{n}$.

Property 1.12. If $J_{n}$ is a flower snark, then the following statements hold:

- The shortest path between any two $b$ vertices leads over $b$ vertices.

- For each pair of a vertices, one shortest path leads over b vertices.

- The shortest path between one a vertex and one $b$ vertex also leads over $b$ vertices.

\section{WEAKLY CONVEX DOMINATION NUMBER AND CONVEX DOMINATION NUMBER FOR GENERALIZED PETERSEN GRAPHS}

In this section we present the results related to the weakly convex domination and convex domination numbers of generalized Petersen graphs. In order to obtain the exact values for these two numbers for graphs of small dimensions, the integer linear programming (ILP) model from [19, with implementation in the CPLEX optimization software package, is used. Since both WCDSP and CDSP are NPhard in a general case, exact algorithms cannot be used to determine solutions for medium-size or large-scale graphs in a reasonable time. However, the ILP model from [19] has been shown to be useful in determining exact solutions for sparse graphs up to 200 vertices.

The following two remarks state some obvious facts for the case $k=1$. 
Remark 2.1. Let $k=1$ and let $G P(n, 1)$ be a generalized Petersen graph. The shortest path between two $u$ vertices in $G P(n, 1)$ consists of only $u$ vertices. Therefore, if two $u$ vertices $u_{i}, u_{j}$ belong to a weakly convex dominating set $S$, then at least one of the sets $\left\{u_{i+1}, u_{i+2}, \ldots, u_{j-1}\right\}$ or $\left\{u_{j+1}, u_{j+2}, \ldots, u_{i-1}\right\}$ belongs to $S$. Notice that the indices are taken modulo $n$.

Remark 2.2 (Corollary of Remark 2.1). Let $k=1$ and let $G P(n, 1)$ be a generalized Petersen graph. If at least one vertex from $u$ vertices belongs to a weakly convex dominating set $S$, then either all $u$ vertices belong to $S$ or a set of $l$, $l \leq\left\lceil\frac{n}{2}\right\rceil$, consecutive $u$ vertices belong to $S$ and the remaining $n-l$ vertices do not belong to $S$.

These two remarks also hold for the $v$ vertices.

The following lemma contains some useful statements for generalized Petersen graphs $G P(n, 3), n \geq 17$.

Lemma 2.3. Let $S$ be a minimal weakly convex dominating set of a generalized Petersen graph $G P(n, 3)$ and $n \geq 17$. Then the following statements hold:

(i) At least one vertex from the set of $v$ vertices belongs to $S$.

(ii) If a vertex $v_{i}$ from $v$ vertices belongs to $S$, then the vertex $v_{i+3}$ belongs to $S$.

(iii) If a vertex $v_{i}$ from $v$ vertices belongs to $S$, then all vertices from the set $\left\{v_{i+3 k} \mid 0 \leq k \leq n-1\right\}$ belong to $S$.

(iv) If a vertex $v_{i}$ from $v$ vertices belongs to $S$ and $n \not \equiv 0(\bmod 3)$, then all $v$ vertices belong to $S$.

(v) If all $v$ vertices belong to $S$, then all $u$ vertices also belong to $S$.

(vi) If all $u$ vertices belong to $S$, then all $v$ vertices also belong to $S$.

Proof.

(i) Suppose that no one $v$ vertex belongs to $S$. Then for each $i, 0 \leq i \leq n-1$, the vertex $v_{i}$ is dominated by $u_{i}$, and therefore $S \supseteq\left\{u_{i} \mid 0 \leq i \leq n-1\right\}$. Consider the vertices $u_{0}$ and $u_{6}$. The unique shortest path between them is of length 4: $u_{0}-v_{0}-v_{3}-v_{6}-u_{6}$, which implies that $v_{0}, v_{3}$, and $v_{6}$ belong to $S$. This is a contradiction with the starting assumption.

(ii) Let $v_{i} \in S$. Consider the vertex $u_{i+6}$ : let it be dominated by a vertex $x$, $x \in S \cap \mathcal{N}\left[u_{i+6}\right]$. There are four cases:

- $x=v_{i+6}$. The unique shortest path $v_{i}-v_{i+3}-v_{i+6}$ is of length 2 and it contains $v_{i+3}$, therefore $v_{i+3} \in S$.

- $x=u_{i+6}$. The unique shortest path $v_{i}-v_{i+3}-v_{i+6}-u_{i+6}$ is of length 3 and it also contains $v_{i+3}$, therefore $v_{i+3} \in S$.

- $x=u_{i+7}$. The unique shortest path $v_{i}-v_{i+3}-v_{i+6}-u_{i+6}-u_{i+7}$ is of length 4. Again, it contains $v_{i+3}$, therefore $v_{i+3} \in S$.

- $x=u_{i+5}$. There are two shortest paths of length 4 in $\operatorname{GP}(n, 3)$ : $v_{i}-v_{i+3}-v_{i+6}-u_{i+6}-u_{i+5}$ and $v_{i}-v_{i+3}-u_{i+3}-u_{i+4}-u_{i+5}$. Both of them contain $v_{i+3}$, and therefore $v_{i+3} \in S$.

(iii) The claim is a consequence of (ii). 
(iv) Since 3 is coprime with $n$, the set $\left\{v_{i+3 k} \mid 0 \leq k \leq n-1\right\}$ is in fact equal to the set of all $v$ vertices, where indices are taken modulo $n$. Thus the claim holds from (iii).

(v) Let $u_{i}$ be an arbitrary vertex from $u$ vertices. The unique shortest path between $v_{i}$ and $v_{i+1}$ is of length $3: v_{i}-u_{i}-u_{i+1}-v_{i+1}$, therefore $u_{i} \in S$.

(vi) Let $v_{i}$ be an arbitrary vertex from $v$ vertices. The unique shortest path between $u_{i}$ and $u_{i+6}$ of length $4: u_{i}-v_{i}-v_{i+3}-v_{i+6}-u_{i+6}$, therefore $v_{i} \in S$.

Let us now present the closed formula for the weakly convex domination number for generalized Petersen graphs.

Theorem 2.4. Let $G P(n, k)$ be a generalized Petersen graph. Then

$$
\gamma_{\text {won }}(G P(n, k))= \begin{cases}2 n, & k \geq 2 \wedge n \geq 13 \\ n, & k=1 \wedge n \neq 3 ; \\ 2, & k=1 \wedge n=3 ; \\ 4, & k=2 \wedge n=5 \\ n, & k=2 \wedge 6 \leq n \leq 11 \\ 24, & k=2 \wedge n=12 ; \\ n, & k=3 \wedge 7 \leq n \leq 10 \\ 22, & k=3 \wedge n=11 \\ 24, & k=3 \wedge n=12 \\ 9, & k=4 \wedge n=9 ; \\ 14, & k=4 \wedge n=10 \\ 22, & k=4 \wedge n=11 \\ 24, & k=4 \wedge n=12 \\ 11, & k=5 \wedge n=11 \\ 18, & k=5 \wedge n=12\end{cases}
$$

Proof. All cases of generalized Petersen graphs $G P(n, k)$, with $(k=1 \wedge n \leq 6) \vee$ $(k=2 \wedge n \leq 31) \vee(k \geq 3 \wedge n \leq 16)$, have a relatively small number of vertices, so they can be checked by the exact method.

Case 1: $k=1, n \geq 7$.

Let $S=\left\{u_{i} \mid 0 \leq i \leq n-1\right\}$. We will prove that $S$ is a weakly convex dominating set of minimum cardinality, i.e. $\gamma_{w c o n}(G P(n, 1))=|S|=n$. It is obvious that $S$ is weakly convex: because of Remark 2.1. the shortest path between two $u$ vertices contains only $u$ vertices (Figure 3). Further, for each vertex $v_{i} \in V \backslash S$, it holds that $v_{i} \in \mathcal{N}\left(u_{i}\right) \wedge u_{i} \in S$, so the set $S$ is also a dominating set.

Suppose that $S^{\prime}$ is a weakly dominating set and $\left|S^{\prime}\right|<n$. Without loss of generality, let us analyze the case when $l, 0<l<n, u$ vertices belong to $S^{\prime}$. Because of Remark 2.2, that is a set of $l$ consecutive vertices. Without loss of generality, let us assign them as $\left\{u_{0}, u_{1}, \ldots, u_{l-1}\right\}$. Since $n \geq 7$, there are at least 


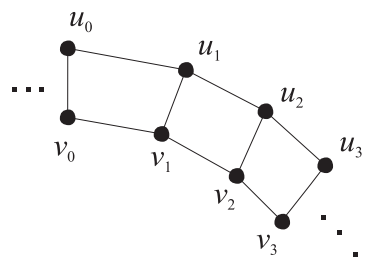

Figure 3. A part of $G P(n, 1)$

three $u$ vertices $\left\{u_{l}, u_{l+1}, \ldots, u_{n-1}\right\}$ which do not belong to $S^{\prime}$. Let us consider the vertex $u_{l+1}$. Since it has to be dominated by a vertex from $S^{\prime}$ and since two $u$ adjacent vertices $u_{l}$ and $u_{l+2}$ are not in $S^{\prime}$ (also $u_{l+1} \notin S^{\prime}$ ), the only remaining option is that $v_{l+1} \in S^{\prime}$. Now consider the shortest paths between two vertices from $S^{\prime}: u_{l-1}$ and $v_{l+1}$. In $G, d_{G}\left(u_{l-1}, v_{l+1}\right)=3$ and there are three shortest paths of length 3: $u_{l-1}-u_{l}-u_{l+1}-v_{l+1}, u_{l-1}-u_{l}-v_{l}-v_{l+1}$, and $u_{l-1}-v_{l-1}-v_{l}-v_{l+1}$. Since $u_{l}, u_{l+1} \notin S^{\prime}$, at least one vertex from the first and the second path is not in $S^{\prime}$; we conclude that all vertices from the latter path belong to $S^{\prime}$, i.e. $v_{l-1}, v_{l} \in S^{\prime}$.

Further, for each $i$ such that $l+2 \leq i \leq n-2, u_{i}$ is dominated by $v_{i}$ since $u_{i-1}, u_{i}, u_{i+1} \notin S^{\prime}$, so $v_{l+2}, \ldots, v_{n-2} \in S^{\prime}$. Again, there are three shortest paths between $u_{0}$ and $v_{n-2}$ of length $3: u_{0}-u_{n-1}-u_{n-2}-v_{n-2}, u_{0}-u_{n-1}-v_{n-1}-v_{n-2}$, and $u_{0}-v_{0}-v_{n-1}-v_{n-2}$. Since $u_{n-1}, u_{n-2} \notin S^{\prime}$, at least one vertex from the first and the second path is not in $S^{\prime}$; we conclude that $v_{0}, v_{n-1} \in S^{\prime}$.

Finally, $\left\{u_{0}, u_{1}, \ldots, u_{l-1}, v_{l-1}, v_{l}, \ldots, v_{n-1}, v_{0}\right\} \subseteq S^{\prime}$, so $\left|S^{\prime}\right| \geq l+n-l+2=n+2$. Therefore, this construction leads to a weakly convex dominating set of a cardinality greater than $n$, which is a contradiction with the assumption $\left|S^{\prime}\right|<n$.

Since the $u$ and $v$ vertices have the same meaning in $\operatorname{GP}(n, 1)$, the analysis when we start with $v$ vertices is analogous.

Case 2: $k=2$ and $n \geq 32$.

Let $m=\left\lfloor\frac{n}{4}\right\rfloor-1$. Let $S$ be a weakly dominating set of $G P(n, 2), n \geq 32$. Let us denote the set of all vertices of $\operatorname{GP}(n, 2)$ by $V$. We will prove that $S=V$, i.e. $\gamma_{w c o n}(G P(n, 2))=2 n$.

Let $v_{i}$ be an arbitrary vertex from $v$ vertices. Since $S$ is a dominating set, we have:

$$
\begin{gathered}
v_{i+2} \in V \Longrightarrow v_{i} \in S \vee v_{i+2} \in S \vee u_{i+2} \in S \vee v_{i+4} \in S ; \\
v_{i+2 m-2} \in V \Longrightarrow v_{i+2 m-4} \in S \vee v_{i+2 m-2} \in S \vee u_{i+2 m-2} \in S \vee v_{i+2 m} \in S
\end{gathered}
$$

Let $x$ be a vertex from the set $\left\{v_{i}, v_{i+2}, u_{i+2}, v_{i+4}\right\}$ and $y$ a vertex from $\left\{v_{i+2 m-4}\right.$, $\left.v_{i+2 m-2}, u_{i+2 m-2}, v_{i+2 m}\right\}$. Although there are many possibilities for vertices $x$ and $y$ satisfying the formulas (2.1) and 2.2), for every case, the unique shortest path between them contains the sub-path $P^{\prime}=v_{i+4}-v_{i+6}-\cdots-v_{i+2 m-6}-v_{i+2 m-4}$ (Figure 4). 


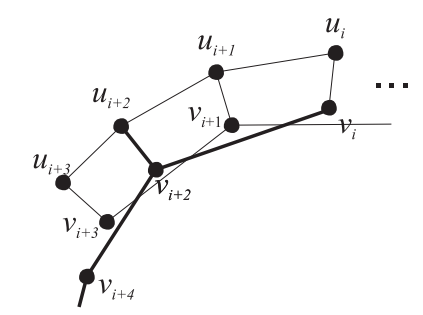

$\cdot$

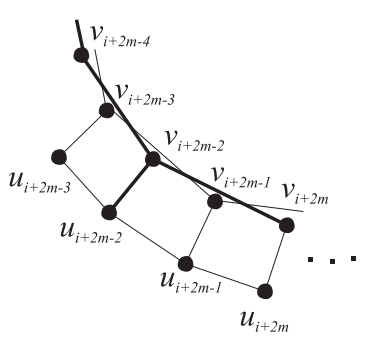

Figure 4. A scheme for constructing the path between $x$ and $y$

Since $x, y \in S$, and $S$ is weakly convex, all vertices from $P^{\prime}$ belong to $S$. For each pair of vertices $\left(v_{i+2}, v_{i+2 m-2}\right), 0 \leq i \leq n-1$, we have

$$
\bigcup_{i=0}^{n-1}\left\{v_{i+4}, v_{i+6}, \ldots, v_{i+2 m-6}, v_{i+2 m-4}\right\}=\left\{v_{j} \mid j=0, \ldots, n-1\right\} \subset S
$$

Therefore, all of the $v$ vertices belong to $S$.

Further, let $i, 0 \leq i \leq n-1$, be an arbitrary index and let us consider the vertex $u_{i}$. Since $v_{i}, v_{i+1} \in S$ and $S$ is weakly convex, the unique shortest path between these two vertices $v_{i}-u_{i}-u_{i+1}-v_{i+1} \in S$ (Figure 5 ). This implies that $u_{i} \in S$, and the proof is finished. 


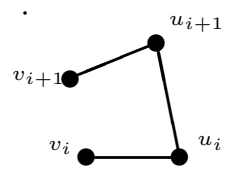

Figure 5. The shortest path between $v_{i}$ and $v_{i+1}$.

Case 3: $k=3$ and $n \geq 17$.

From Lemma 2.3 (i), at least one vertex from $v$ vertices belong to $S$. Without loss of generality, let $v_{0}$ be that vertex. We consider two cases:

(a) $n \not \equiv 0(\bmod 3)$. From Lemma 2.3 (iv) and (v), all $v$ vertices and all $u$ vertices belong to $S$. Therefore, $|S|=2 n$.

(b) $n \equiv 0(\bmod 3)$. From Lemma 2.3 (iii) we have that $\left\{v_{3 i} \mid 0 \leq i \leq n / 3-\right.$ $1\} \subset S$. We will show that at least one more vertex from $v$ vertices belongs to $S$.

Suppose that this is not true, i.e. all other $v$ vertices are not in $S$. Since the vertex $v_{i} \notin S$ has neighbourhood $\mathcal{N}\left(v_{i}\right)=\left\{v_{i-3}, u_{i}, v_{i+3}\right\}$ and $v_{i-3}, v_{i+3} \notin S, v_{i}$ must be dominated by $u_{i} \in S$. Therefore, $\left\{u_{3 k+1}, u_{3 k+2} \mid\right.$ $0 \leq k<n / 3\} \subset S$. Since for an arbitrary $k$ the unique shortest path between $u_{3 k+2}$ and $u_{3(k+1)+1}$ is of length 2 and contains $u_{3(k+1)}$, we conclude that the rest of $u$ vertices, i.e. the set $\left\{u_{3 k} \mid 0 \leq k \leq n-1\right\}$, is a subset of $S$. From Lemma 2.3 (vi), we have that all $v$ vertices belong to $S$, which is a contradiction with the assumption that other $v$ vertices are not in $S$.

Therefore, at least one more $v$ vertex belongs to $S$. Without loss of generality, let $v_{1}$ be that vertex. From Lemma 2.3 (iii), we have that $\left\{v_{3 k+1} \mid 0 \leq k \leq n / 3-1\right\}$ is a subset of $S$. Let us consider pairs of successive $v$ vertices $v_{3 k}, v_{3 k+1}, 0 \leq k<n / 3$, belonging to $S$. The unique shortest path between the vertices from each pair is $v_{3 k}-u_{3 k}-u_{3 k+1}-v_{3 k+1}$; therefore $u_{3 k}, u_{3 k+1} \in S$, for $0 \leq k<n / 3$. Similarly, as in the previous paragraph, we further conclude that the rest of $u$ vertices belong to $S$, and because of Lemma 2.3 (vi), we have that all $v$ vertices also belong to $S$. Therefore $|S|=2 n$. 
Case 4: $k \geq 4$ and $n \geq 17$.

Let $S$ be a weakly convex dominating set. Let us first show that all $u$ vertices belong to $S$.

Suppose that a $u$ vertex, say $u_{i}$, is not in $S$. Since $u_{i}$ has to be dominated, we have three cases: $u_{i-1}$ or $v_{i}$ or $u_{i+1}$ belong to $S$.

Suppose that $u_{i-1} \in S$. Then $u_{i+1} \notin S$, since the unique shortest path in $G$ between $u_{i-1}$ and $u_{i+1}$ is of length 2 and it contains $u_{i}$. Since $u_{i+1}$ has to be dominated, then either $v_{i+1}$ or $u_{i+2}$ belong to $S$. However, the unique shortest path in $G$ between $u_{i-1}$ and $v_{i+1}$, as well as between $u_{i-1}$ and $u_{i+2}$, is of length 3 and contains vertices $u_{i}$ and $u_{i+1}$. Since $S$ is weakly convex and the shortest path is unique, $u_{i}$ must belong to $S$, which contradicts the assumption $u_{i} \notin S$.

The case $u_{i+1} \in S$ is symmetric to the previous case $u_{i-1} \in S$.

Suppose that $v_{i} \in S$. Again, $u_{i+1} \notin S$, since the unique shortest path in $G$ between $v_{i}$ and $u_{i+1}$ is of length 2 and it contains $u_{i}$. Since $u_{i} \notin S, u_{i+1}$ is dominated either by $v_{i+1}$ or by $u_{i+2}$. The unique shortest path in $G$ between $v_{i}$ and $v_{i+1}$ is $v_{i}-u_{i}-u_{i+1}-v_{i+1}$, and that between $v_{i}$ and $u_{i+2}$ is $v_{i}-u_{i}-u_{i+1}-u_{i+2}$. Both paths contain $u_{i}$, which contradicts the starting assumption.

Therefore, all $u$ vertices belong to $S$. Consider now vertices $u_{i}$ and $u_{i+k}$. The unique shortest path in $G$ between them is of length 3: $u_{i}-v_{i}-v_{i+k}-u_{i+k}$. Since $S$ is weakly convex, vertices from that path must belong to $S$, i.e. $v_{i}, v_{i+k} \in S$. For each pair of vertices $\left(u_{i}, u_{i+k}\right), 0 \leq i \leq n-1$, we have

$$
\bigcup_{i=0}^{n-1}\left\{v_{i}, v_{i+k}\right\}=\left\{v_{j} \mid 0 \leq j \leq n-1\right\} \subset S .
$$

We have proved that all $v$ vertices also belong to $S$ and $\gamma_{w c o n} G P(n, k)=|S|=$ $2 n$.

Corollary 2.5. Let $G P(n, k)$ be a generalized Petersen graph. Then

$$
\gamma_{\text {con }}(G P(n, k))= \begin{cases}n, & k=1 \wedge n \geq 4 \\ 2 n, & k \geq 2 \wedge n \geq 8 \\ 2, & k=1 \wedge n=3 \\ 4, & k=2 \wedge n=5 \\ 6, & k=2 \wedge n=6 \\ 7, & n=7\end{cases}
$$

Proof. Let $k=1$. Cases $n \in\{3,4,5\}$ were checked by the exact method. Let $n \geq 6$. Since $G P(n, 1) \equiv C_{n} \square P_{2}$ (Remark 1.8) and $\gamma_{\text {con }}\left(C_{n}\right)=n$ (Remark 1.4 for $n \geq 6$, from Theorem 1.7 it follows that, for $n \geq 6$, the convex domination number $\gamma_{\text {con }}(G P(n, 1))=\gamma_{\text {con }}\left(C_{n} \square P_{2}\right)=\min \{n \cdot 1,2 \cdot n\}=n$.

Let $k \geq 2$. Since $\gamma_{w c o n}(G P(n, k)) \leq \gamma_{c o n}(G P(n, k))$, Theorem 2.4 finishes the proof for larger values of $n$. The formula for the convex domination number for smaller graphs can be checked by the exact method. 


\section{WEAKLY CONVEX DOMINATION NUMBER AND CONVEX DOMINATION NUMBER FOR FLOWER SNARK GRAPHS}

In this section we propose closed formulas for the convex domination number and the weakly convex domination number for flower snark graphs.

3.1. The convex domination number for flower snark graphs. If $n=5$, by the exact method it can be easily checked that $\gamma_{\text {con }}\left(J_{5}\right)=20$. If $n \geq 7$, we have that the length of the shortest cycle of $J_{n}$ is 6 . Since $\delta\left(J_{n}\right)=3 \geq 2$, the conditions of Theorem 1.5 are satisfied, so the following proposition holds.

Proposition 3.1. For every flower snark $J_{n}$ where $n \geq 7$ is an odd number, it holds that $\gamma_{\text {con }}\left(J_{n}\right)=4 n$.

3.2. The weakly convex domination number for flower snark graphs. If $n=5$, one can easily check that $\gamma_{w c o n}\left(J_{5}\right)=10$ by the exact method. Therefore, in the following theorem, we suppose that $n \geq 7$.

Theorem 3.2. For every flower snark $J_{n}$ where $n \geq 7$ is an odd number, it holds that $\gamma_{w c o n}\left(J_{n}\right)=2 n$.

Proof. The proof is organized in three steps.

\section{Step 1.}

Let $S$ be a set containing all $a$ and all $b$ vertices, i.e., $S=\left\{a_{i} \mid 0 \leq i \leq\right.$ $n-1\} \cup\left\{b_{i} \mid 0 \leq i \leq n-1\right\}$. We will show that $S$ is a weakly convex dominating set with minimal cardinality. Obviously, $S$ is a dominating set, since for each $i$, $0 \leq i \leq n-1$, vertices $c_{i}$ and $d_{i}$ are dominated by $a_{i}$. Further, from Property 1.12 one can conclude that $S$ is a weakly convex set.

Let us now suppose that there is a weakly convex dominating set $S^{\prime}$ such that $\left|S^{\prime}\right|<2 n$.

\section{Step 2.}

We first show that all $b$ vertices must belong to $S^{\prime}$.

Suppose that there is a $b$ vertex not belonging to $S^{\prime}$. We consider two cases:

(a) $(\exists i \in\{0,1, \ldots, n-1\}) b_{i} \in S^{\prime}$ and $(\exists j \in\{0,1, \ldots, n-1\}) b_{j} \notin S^{\prime}$.

(b) $(\forall i \in\{0,1, \ldots, n-1\}) b_{i} \notin S^{\prime}$.

Case (a). Since there are $b$ vertices which belong and $b$ vertices which do not belong to $S^{\prime}$, we can assume that there are two adjacent $b$ vertices such that one belongs to $S^{\prime}$ and the other does not. Because of Property 1.10 suppose, without loss of generality, that $b_{0} \notin S^{\prime}$ and $b_{n-1} \in S^{\prime}$. Then, because of Property $1.1 . b_{1} \notin$ $S^{\prime}$ and $b_{2} \notin S^{\prime}$, since the unique shortest path between $b_{n-1}$ and $b_{1}$ (respectively $b_{2}$ ) leads via $b_{0}$ (which is not in $S^{\prime}$ ). Then, the vertex $a_{1}$ must belong to $S$ to dominate $b_{1}$. However, the shortest path between $b_{n-1}$ and $a_{1}$ leads via $b_{0}$ and $b_{1}$, so $b_{0} \in S$, which is a contradiction with $b_{0} \notin S^{\prime}$.

Case (b). Let no $b$ vertex belong to $S^{\prime}$. Then for each $i, 0 \leq i \leq n-1$, the vertex $b_{i}$ is dominated by the vertex $a_{i}$, so all $a$ vertices belong to $S^{\prime}$. Let us consider the shortest paths between vertices $a_{0}$ and $a_{1}$. There are two possible shortest paths between these two vertices and (at least) one of them must belong to $S^{\prime}$ : 
(i) $a_{0}-c_{0}-c_{1}-a_{1}$.

(ii) $a_{0}-d_{0}-d_{1}-a_{1}$.

It should be noted that the shortest path $a_{0}-b_{0}-b_{1}-a_{1}$ is not possible, since $b$ vertices do not belong to $S^{\prime}$.

Subcase (i). Consider two vertices $c_{1}$ and $a_{2}$ both of which belong to $S$. The unique shortest path between $c_{1}$ and $a_{2}$ is of length 2: $c_{1}-c_{2}-a_{2}$, therefore $c_{2} \in S^{\prime}$. Now, the shortest path between $c_{2}$ and $a_{3}$ is of length 2: $c_{2}-c_{3}-a_{3}$, so we conclude that $c_{3} \in S^{\prime}$. By using the isomorphism defined in Property 1.9 and applying the same argumentation, we can prove that for each $i, 1 \leq i \leq n-4$, the vertex $c_{i+3}$ belongs to $S^{\prime}$. Since all $a$ vertices also belong to $S^{\prime}$, we get that $\left|S^{\prime}\right| \geq n+n=2 n$, which is a contradiction with the fact that $\left|S^{\prime}\right|<2 n$.

Subcase (ii). Analogously to the Subcase (i), it can be proved that $\left|S^{\prime}\right| \geq 2 n$, which is a contradiction with the fact that $\left|S^{\prime}\right|<2 n$.

Since we proved that none of the possible shortest paths between $a_{0}$ and $a_{1}$ can not belong to $S^{\prime}$, we proved that the Case (b) leads to a contradiction.

Therefore, all $b$ vertices belong to $S^{\prime}$. 


\section{Step 3.}

Since $\left|S^{\prime}\right|<2 n$ and all $b$ vertices belong to $S^{\prime}$, at least one $a$ vertex does not belong to $S^{\prime}$.

Without loss of generality, suppose that $a_{2} \notin S^{\prime}$. Then, from Property 1.1, we also get that $c_{2} \notin S^{\prime}$, since the unique shortest path between $b_{2}$ and $c_{2}$ leads via $a_{2}$. The vertex $c_{2}$ must be dominated by a vertex from $S^{\prime}$. Since $a_{2}, c_{2} \notin S^{\prime}$, we have two remaining options: either $c_{1}$ or $c_{3}$ must belong to $S^{\prime}$.

Case 1: $c_{1} \in S^{\prime}$. Then the vertex $c_{3}$ is not dominated by any vertex from $S^{\prime}$, because:

(i) The unique shortest path between $c_{1}$ and $c_{3}$, i.e. $c_{1}-c_{2}-c_{3}$, leads via $c_{2}$, which is not in $S^{\prime}$. From Property 1.1 we conclude that $c_{3} \notin S^{\prime}$.

(ii) $c_{2} \notin S^{\prime}$, which was already stated.

(iii) The unique shortest path between $c_{1}$ and $c_{4}$ is $c_{1}-c_{2}-c_{3}-c_{4}$. Since $c_{2} \notin S^{\prime}$, from Property 1.1 we get that $c_{4} \notin S^{\prime}$.

(iv) The unique shortest path between $c_{1}$ and $a_{3}$, which is $c_{1}-c_{2}-c_{3}-a_{3}$, leads via $c_{2}$ which is not in $S^{\prime}$. From Property 1.1 we conclude that $a_{3} \notin S^{\prime}$.

Thus, we get a contradiction with the starting assumption that $S^{\prime}$ is a weakly convex dominating set and $\left|S^{\prime}\right|<2 n$.

Case 2: $c_{3} \in S^{\prime}$. Analogously to the Case 1 , it can be proved that the vertex $c_{1}$ is not dominated by any vertex from $S^{\prime}$. This is a contradiction with the assumption that $S^{\prime}$ is a weakly convex dominating set and $\left|S^{\prime}\right|<2 n$.

Finally, we conclude that $\gamma_{w c o n}\left(J_{n}\right)=2 n$.

\section{Conclusions}

In this paper, the weakly convex domination number and convex domination number for the generalized Petersen graphs and flower snark graphs are studied. We prove that in the case of the generalized Petersen graph $\operatorname{GP}(n, k)$, for $k \geq 2$ and $n \geq 13$, both minimal weakly convex dominating set and minimal convex dominating sets must contain all vertices. For $k=1$ and $n \geq 4$, both minimal weakly convex domination number and convex domination number are equal to $n$. We also obtain the closed formula for the weakly convex domination number and the convex domination number for flower snark graphs.

This work can be extended in several directions. It could be useful to determine the closed formula for calculating the weakly convex and/or convex domination numbers for some other challenging classes of graphs. Since the problems are NPhard in a general case, determining the exact solutions for large-scale graphs is not possible in a reasonable time. This fact opens the possibility of attacking the problem via some heuristic approach.

\section{REFERENCES}

[1] S. S. Adams et al., The minimum span of $L(2,1)$-labelings of certain generalized Petersen graphs, Discrete Appl. Math. 155 (2007), no. 10, 1314-1325. MR 2332323.

[2] S. Ahmad et al., On the metric dimension of generalized Petersen graphs, Quaest. Math. 36 (2013), no. 3, 421-435. MR 3176029 
[3] A. Behzad, M. Behzad and C. E. Praeger, On the domination number of the generalized Petersen graphs, Discrete Math. 308 (2008), no. 4, 603-610. MR 2376483

[4] B. Behsaz, P. Hatami and E. S. Mahmoodian, On minimum vertex covers of generalized Petersen graphs, Australas. J. Combin. 40 (2008), 253-264. MR 2381433.

[5] B. Brešar and T. Kraner Šumenjak, On the 2-rainbow domination in graphs, Discrete Appl. Math. 155 (2007), no. 17, 2394-2400. MR 2360671

[6] C. N. Campos, S. Dantas and C. P. de Mello, The total-chromatic number of some families of snarks, Discrete Math. 311 (2011), no. 12, 984-988. MR 2787309

[7] H. S. M. Coxeter, Self-dual configurations and regular graphs, Bull. Amer. Math. Soc. 56 (1950), 413-455. MR 0038078

[8] J. Cyman, M. Lemańska and J. Raczek, Graphs with convex domination number close to their order, Discuss. Math. Graph Theory 26 (2006), no. 2, 307-316. MR 2330331.

[9] M. Dettlaff and M. Lemańska, Influence of edge subdivision on the convex domination number, Australas. J. Combin. 53 (2012), 19-30. MR 2962128.

[10] D. Ferrero and S. Hanusch, Component connectivity of generalized Petersen graphs, Int. J. Comput. Math. 91 (2014), no. 9, 1940-1963. MR 3264870

[11] J. Fox, R. Gera and P. Stănică, The independence number for the generalized Petersen graphs, Ars Combin. 103 (2012), 439-451. MR 2907353.

[12] X. Fu, Y. Yang and B. Jiang, On the domination number of generalized Petersen graphs $P(n, 2)$, Discrete Math. 309 (2009), no. 8, 2445-2451. MR 2509012

[13] L. Gao et al., The decycling number of generalized Petersen graphs, Discrete Appl. Math. 181 (2015), 297-300. MR 3284536.

[14] M. Ghebleh et al., The circular chromatic index of flower snarks, Electron. J. Combin. 13 (2006), no. 1, Note 20, 7 pp. MR 2274302

[15] Kh. Md. M. Haque et al., Prime cordial labeling of flower snark and related graphs, Ars Combin. 105 (2012), 45-52. MR 2976356

[16] R. Isaacs, Infinite families of nontrivial trivalent graphs which are not Tait colorable, Amer. Math. Monthly 82 (1975), 221-239. MR 0382052

[17] B. Javad Ebrahimi, N. Jahanbakht and E. S. Mahmoodian, Vertex domination of generalized Petersen graphs, Discrete Math. 309 (2009), no. 13, 4355-4361. MR 2519173

[18] J. Kratica, V. Kovačević-Vujčić and M. Čangalović, The strong metric dimension of some generalized Petersen graphs, Appl. Anal. Discrete Math. 11 (2017), no. 1, 1-10. MR 3648651.

[19] J. Kratica et al., An integer linear programming formulation for the convex domination set problems, preprint, 2019. arXiv:1904.02541 [math.OC].

[20] A. Kucieńska, M. Lemańska and J. Raczek, Weakly convex and convex domination numbers of some products of graphs, Ars Combin. 124 (2016), 409-420. MR 3468826

[21] M. A. Labendia and S. R. Canoy, Jr., Convex domination in the composition and Cartesian product of graphs, Czechoslovak Math. J. 62(137) (2012), no. 4, 1003-1009. MR 3010253.

[22] M. Lemańska, Weakly convex and convex domination numbers, Opuscula Math. 24 (2004), no. 2, 181-188. MR 2100881

[23] M. Lemańska, Nordhaus-Gaddum results for weakly convex domination number of a graph, Discuss. Math. Graph Theory 30 (2010), no. 2, 257-263. MR 2683046

[24] M. Lemańska, J. A. Rodríguez-Velázquez and I. Gonzalez Yero, Nordhaus-Gaddum results for the convex domination number of a graph, Period. Math. Hungar. 65 (2012), no. 1, 125-134. MR 2970070. 
[25] X. Lin et al., The crossing numbers of generalized Petersen graphs with small order, Discrete Appl. Math. 157 (2009), no. 5, 1016-1023. MR 2500719

[26] J. Liu and X. Zhang, The exact domination number of generalized Petersen graphs $P(n, k)$ with $n=2 k$ and $n=2 k+2$, Comput. Appl. Math. 33 (2014), no. 2, 497-506. MR 3217575

[27] S. Naz et al., On the constant metric dimension of generalized Petersen graphs $P(n, 4)$, Acta Math. Sin. (Engl. Ser.) 30 (2014), no. 7, 1145-1160. MR 3226764

[28] O. Ore, Theory of graphs, American Mathematical Society Colloquium Publications, Vol. XXXVIII, American Mathematical Society, Providence, RI, 1962. MR 0150753

[29] J. Raczek, NP-completeness of weakly convex and convex dominating set decision problems, Opuscula Math. 24 (2004), no. 2, 189-196. MR 2100882.

[30] J. Raczek and M. Lemańska, A note on the weakly convex and convex domination numbers of a torus, Discrete Appl. Math. 158 (2010), no. 15, 1708-1713. MR 2671549

[31] C. Tong et al., 2-rainbow domination of generalized Petersen graphs $P(n, 2)$, Discrete Appl. Math. 157 (2009), no. 8, 1932-1937. MR 2514610

[32] G. Xu and L. Kang, On the power domination number of the generalized Petersen graphs, J. Comb. Optim. 22 (2011), no. 2, 282-291. MR 2817954

[33] B. Zelinka, Domination in generalized Petersen graphs, Czechoslovak Math. J. 52(127) (2002), no. 1, 11-16. MR 1885452

[34] E. Zhu et al., Acyclic 3-coloring of generalized Petersen graphs, J. Comb. Optim. 31 (2016), no. 2, 902-911. MR 3449438.

\section{Jozef Kratica}

Mathematical Institute, Serbian Academy of Sciences and Arts, Kneza Mihaila 36/III, 11000

Belgrade, Serbia

jkratica@gmail.com

Dragan Matić凶

Faculty of Natural Sciences and Mathematics, Banja Luka, Bosnia and Herzegovina

dragan.matic@pmf .unibl.org

Vladimir Filipović

Faculty of Mathematics, University of Belgrade, Studentski trg 16/IV, 11000 Belgrade, Serbia

vladofilipovic@hotmail.com

Received: January 9, 2018

Accepted: August 27, 2019 